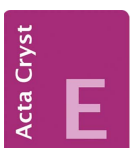

CRYSTALLOGRAPHIC COMMUNICATIONS

ISSN 2056-9890

Received 24 July 2020

Accepted 28 July 2020

Edited by M. Weil, Vienna University of

Technology, Austria

Keywords: $\mathrm{KAIP}_{2} \mathrm{O}_{7}$ structure type; pyrophosphate; scandium; isotypism; structure determination; crystal structure.

CCDC reference: 2019936

Supporting information: this article has supporting information at journals.iucr.org/e

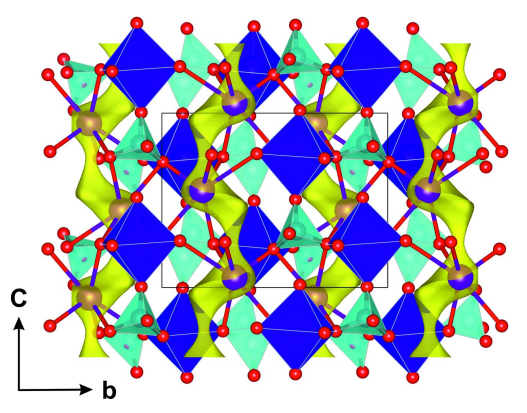

OPEN $\partial$ ACCESS

\section{The crystal structure of $\mathrm{KScP}_{2} \mathrm{O}_{7}$}

\author{
Günther J. Redhammer* and Gerold Tippelt
}

Chemistry and Physics of Materials, University of Salzburg, Jakob-Haringerstr. 2A, 5020 Salzburg, Austria. *Correspondence e-mail: guenther.redhammer@sbg.ac.at

Single crystals of $\mathrm{KScP}_{2} \mathrm{O}_{7}$, potassium scandium diphosphate, were grown in a borate flux. The title compound crystallizes isotypically with $\mathrm{KAlP}_{2} \mathrm{O}_{7}$ in spacegroup type $P 2{ }_{1} / c, Z=4$. The main building block is an $\left\{\mathrm{ScP}_{2} \mathrm{O}_{11}\right\}^{9-}$ unit, forming layers parallel to (001). These layers are stacked along [001] via common corners of octahedral and tetrahedral units to span up large heptagonal cavities that host the potassium cations with a coordination number of 10 . The $\mathrm{P}-\mathrm{O}-\mathrm{P}$ bridging angle increases with increasing size of the octahedrally coordinated $M^{\mathrm{III}}$ cation, as do the $\mathrm{K}-\mathrm{O}$ distances within a series of $\mathrm{KM}^{\mathrm{III}} \mathrm{P}_{2} \mathrm{O}_{7}$ compounds $\left(M^{\mathrm{III}}=\mathrm{Al}\right.$ to $\mathrm{Y}$ with ionic radii $r=0.538$ to $0.90 \AA$ ).

\section{Chemical context}

Metal-phosphates with open framework structures raise large interest due to a rich crystal chemistry (Clearfield, 1988) and possible interesting applications, e.g. as non-linear optical materials, solid-state electrolytes, ionic conductors, battery materials or sensors (Hagerman \& Poeppelmeier, 1995; Vitiņš et al., 2000). In the context of solid-state electrolytes, we recently investigated the Na-super ionic conducting NaSICON-type compounds $\mathrm{Na}_{3} \mathrm{Sc}_{2}\left(\mathrm{PO}_{4}\right)_{3} \quad$ (NSP) and $\mathrm{Ag}_{3} \mathrm{Sc}_{2}\left(\mathrm{PO}_{4}\right)_{3}$ (ASP) in terms of their structural phase-transition sequences and ionic conductivities (Rettenwander et al., 2018; Ladenstein et al., 2020; Redhammer et al., 2020). To elucidate the role of the alkali metals on symmetry, we intended to synthesize the potassium analogue of NSP with flux-growth techniques. Using a method applied by Sljukic et al. (1967) for the synthesis of large crystals of NaSICON-type $\mathrm{KZr}_{2}\left(\mathrm{PO}_{4}\right)_{3}$, however, did not yield the intended compound $\mathrm{K}_{3} \mathrm{Sc}_{2}\left(\mathrm{PO}_{4}\right)_{3}$, but the title diphosphate $\mathrm{KScP}_{2} \mathrm{O}_{7}$ instead.

Vitinsš et al. (2000) reviewed that for such $A^{\mathrm{I}} M^{\mathrm{III}} \mathrm{P}_{2} \mathrm{O}_{7}(A=$ $\mathrm{Li}, \mathrm{Na}, \mathrm{K}, \mathrm{Rb}, \mathrm{Cs}, \mathrm{Tl} ; M=\mathrm{Al}, \mathrm{Ga}, \mathrm{Fe}, \mathrm{In}, \mathrm{Sc}, \ldots$ ) compounds six different structure types can be distinguished. They confirm that structure type I, involving compounds with large $A$ site cations, is the largest group, showing $P 2_{1} / c$ space-group symmetry. $\mathrm{KAlP}_{2} \mathrm{O}_{7}(\mathrm{Ng} \&$ Calvo, 1973) is regarded as the aristo-structure of group I with around 45 different compositions as compiled in the Inorganic Crystal Structure Database (ICSD; Zagorac et al., 2019). For K-containing compounds, further materials are $\mathrm{KCrP}_{2} \mathrm{O}_{7}$ (Gentil et al., 1997), $\mathrm{KGaP}_{2} \mathrm{O}_{7}$ (Genkin \& Timofeeva, 1989), $\mathrm{KFeP}_{2} \mathrm{O}_{7}$ (Riou et al., 1988; Genkin \& Timofeeva, 1989), $\mathrm{KVP}_{2} \mathrm{O}_{7}$ (Benhamada et al., 1991), $\mathrm{KTiP}_{2} \mathrm{O}_{7}$ (Zatovsky et al., 2000), $\mathrm{KMoP}_{2} \mathrm{O}_{7}$ (Leclaire et al., 1989; Chen et al., 1989), $\mathrm{KInP}_{2} \mathrm{O}_{7}$ (Zhang et al., 2004), $\mathrm{KLuP}_{2} \mathrm{O}_{7}$ (Yuan et al., 2007), $\mathrm{KYbP}_{2} \mathrm{O}_{7}$ (Horchani-Naifer \& Férid, 2007), $\mathrm{KErP}_{2} \mathrm{O}_{7}$ (Chaker et al., 2016) and $\mathrm{KYP}_{2} \mathrm{O}_{7}$ (Yuan et al., 2007). Synthesis and lattice parameters of $\mathrm{Ce}^{\mathrm{III}}$ doped polycrystalline $\mathrm{KScP}_{2} \mathrm{O}_{7}$ as well as the luminescence 
properties were reported recently by Zhang et al. (2016); however, no atomic coordinates were given.

In this contribution, we present the determination of the crystal structure of $\mathrm{KScP}_{2} \mathrm{O}_{7}$, not reported so far, and compare it with the series of other K-containing diphosphates.

\section{Structural commentary}

The title compound crystallizes in space group $P 2_{1} / c$ and is isostructural with $\mathrm{KAlP}_{2} \mathrm{O}_{7}(\mathrm{Ng} \&$ Calvo, 1973). It contains one distinct $\mathrm{K}$ and $\mathrm{Sc}$ atom site, two distinct $\mathrm{P}$ atom and seven different oxygen-atom positions, all of them on general position $4 e$. The basic building unit is a pyrophosphate group, which is formed by two distinct $\mathrm{PO}_{4}$ tetrahedra (Fig. 1). They share the $\mathrm{O} 4$ oxygen atom, and the bridging $\mathrm{P} 1-\mathrm{O} 4$ and $\mathrm{P} 2-$ $\mathrm{O} 4$ bond lengths are distinctly longer [1.6128(6) and 1.6076 (6) $\AA$, respectively] than the three shorter terminal PO bonds [ranging between 1.4944 (7) and 1.5207 (6) $\AA$ ]. These latter distances are those to the oxygen atoms which are shared with the $\mathrm{ScO}_{6}$ octahedra. The tetrahedral $\mathrm{O}-\mathrm{P}-\mathrm{O}$ angles involving the bridging oxygen atom $\mathrm{O} 4$ are generally smaller, those involving the terminal oxygen atoms distinctly larger than the ideal $\mathrm{O}-T-\mathrm{O}$ angle of $109.5^{\circ}$. This - together with the difference in bond lengths between bridging and nonbridging $T-\mathrm{O}$ bonds - induces polyhedral distortion (especially for the tetrahedral angle variance, TAV), which is distinctly larger for the P1 tetrahedron. Likewise, the average bond length is slightly larger for the $\mathrm{P} \mathrm{O}_{4}$ tetrahedron than for the $\mathrm{P}_{2} \mathrm{O}_{4}$ tetrahedron (Table 1). When comparing average

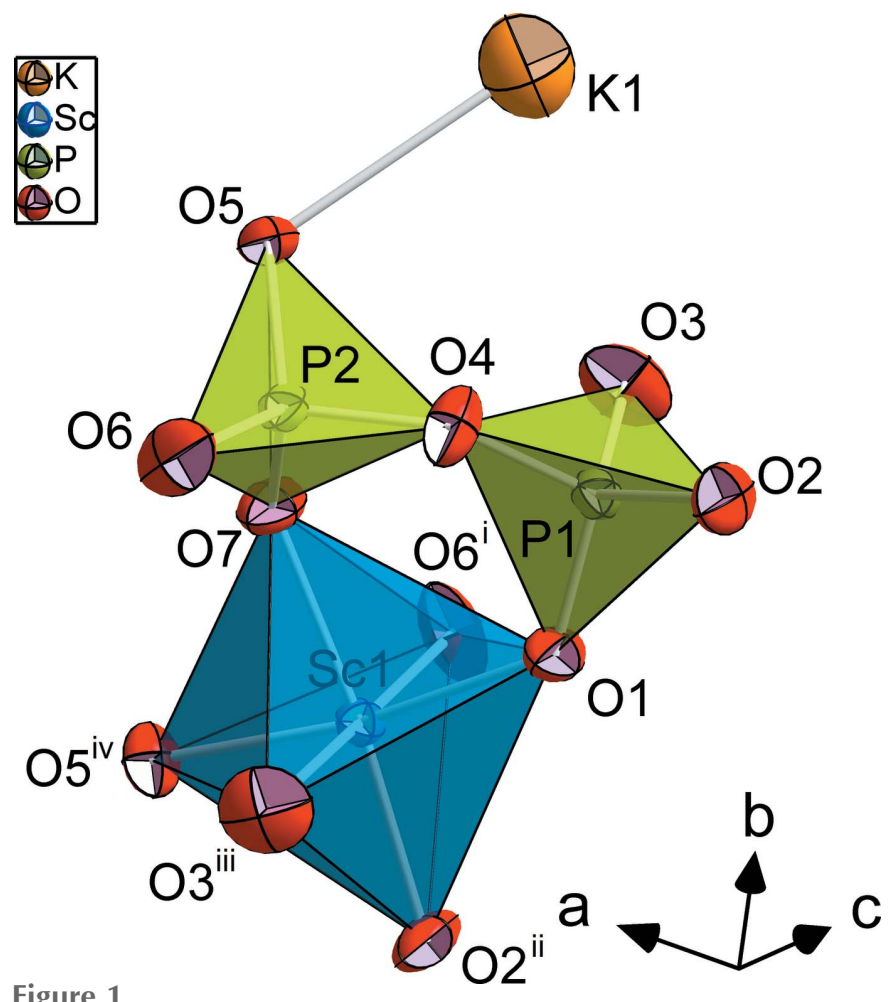

Figure 1

The principal building unit of $\mathrm{KScP}_{2} \mathrm{O}_{7}$ shown with displacement ellipsoids at the $95 \%$ probability level. [Symmetry codes: (i) $x, \frac{1}{2}-y$, $\frac{1}{2}+z$; (ii) $1-x,-\frac{1}{2}+y, \frac{3}{2}-z$; (iii) $x, \frac{1}{2}-y,-\frac{1}{2}+z$; (iv) $\left.2-x,-\frac{1}{2}+y, \frac{3}{2}-z\right]$. bond lengths and polyhedral distortion parameters of the series of $\mathrm{K}^{\mathrm{III}} \mathrm{P}_{2} \mathrm{O}_{7}$ structures $(M=\mathrm{Al}$ to $\mathrm{Y})$, no clear variations with the ionic radius of the $M$ cations can be found from the available data for tetrahedral structure units and distortion parameters, and they remain almost constant. The parameters for $\mathrm{KScP}_{2} \mathrm{O}_{7}$ fit well into the data of the other $\mathrm{KM}^{\mathrm{III}} \mathrm{P}_{2} \mathrm{O}_{7}$ structures. The tetrahedral bridging angle $\mathrm{P} 1-$ $\mathrm{O} 4-\mathrm{P} 2$ amounts to $125.80(5)^{\circ}$ and is distinctly larger than that of $\mathrm{KAlP}_{2} \mathrm{O}_{7}\left[123.2(11)^{\circ}\right]$. On the other hand, here a clear trend of increasing bridging angle with increasing size of the $M$ cation is evident, i.e. the pyrophosphate group is stretched to account for the increase in size of the $M$ cations (Table 1 ).

The terminal oxygen atoms of the pyrophosphate group share their corners with five neighbouring $\mathrm{ScO}_{6}$ octahedra. Following Leclaire et al. (1989), two phosphate tetrahedra and one octahedron form the basic $\left\{\mathrm{ScP}_{2} \mathrm{O}_{11}\right\}^{9-}$ units ( $c f$. Fig. 1), which are connected with units of the same kind via cornersharing to make up a sheet parallel to (001), as depicted in Fig. 2. These layers are stacked along [001] in such a way that a

(a)
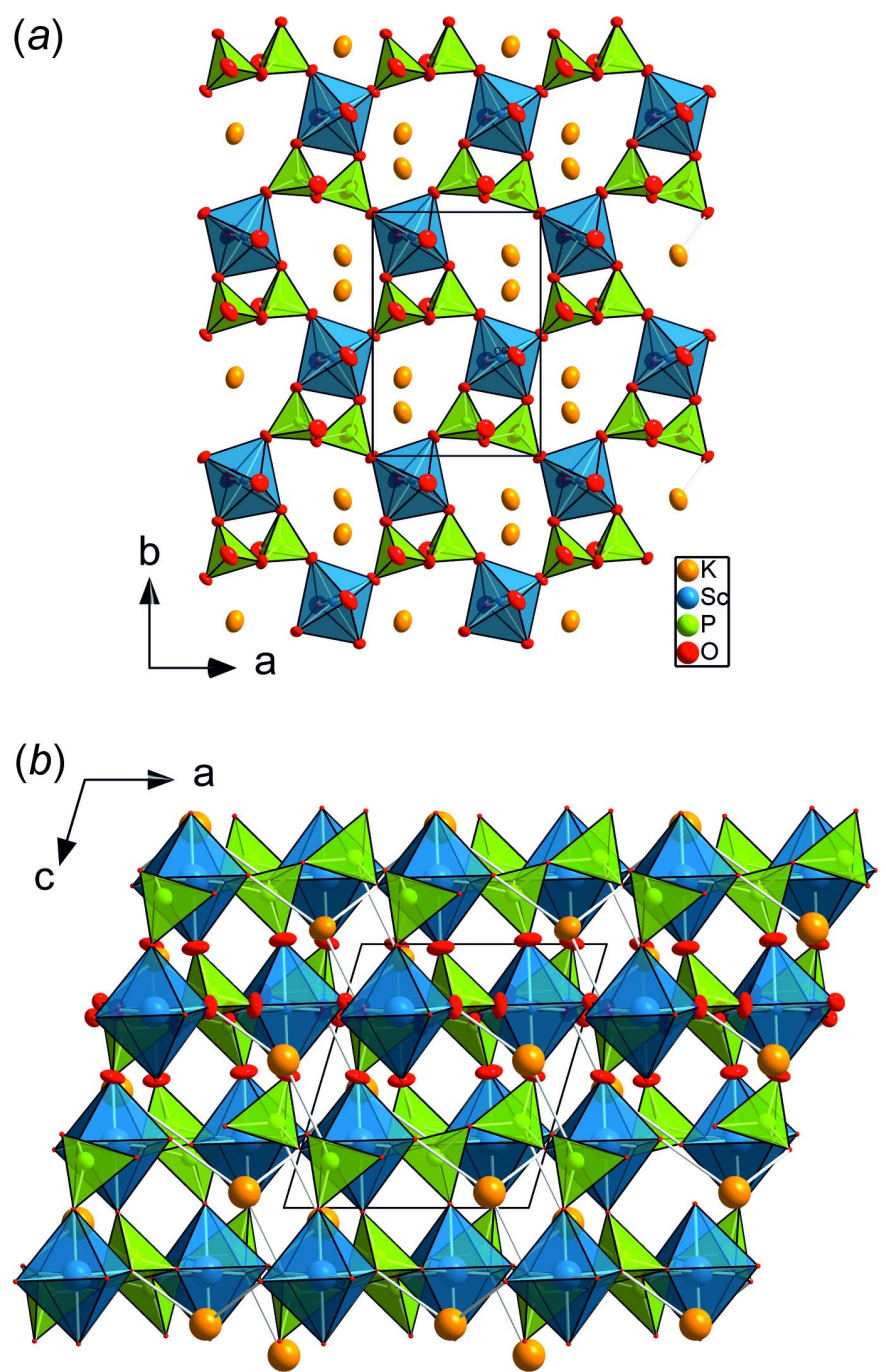

Figure 2

(a) Layer of laterally interconnected $\left\{\mathrm{ScP}_{2} \mathrm{O}_{11}\right\}^{9-}$ units, forming a layer parallel to $(001)$, the $\mathrm{K}^{\mathrm{I}}$ cations are hosted in the channels extending along [001]; (b) the three-dimensional framework structure of $\mathrm{KScP}_{2} \mathrm{O}_{7}$, viewed along [010]. 
Table 1

Selected structural and distortional parameters $\left(\AA{ }^{\circ},{ }^{\circ}\right)$ of $\mathrm{KM}^{\mathrm{III}} \mathrm{P}_{2} \mathrm{O}_{7}$ compounds.

Vol. = polyhedral volume $\left(\AA^{3}\right)$, DI = distortion index, ECoN = effective coordination number, OQE = octahedral quadratic elongation, OAV $=$ octahedral angle variance $\left({ }^{\circ}\right), T Q E=$ tetrahedral quadratic elongation, TAV $=$ tetrahedral angle variance $\left({ }^{\circ}\right)$. All calculations were performed using $V E S T A($ Momma $\&$ Izumi 2011; for the mathematical meaning see the VESTA Handbook); ionic radii were taken from Shannon (1976).

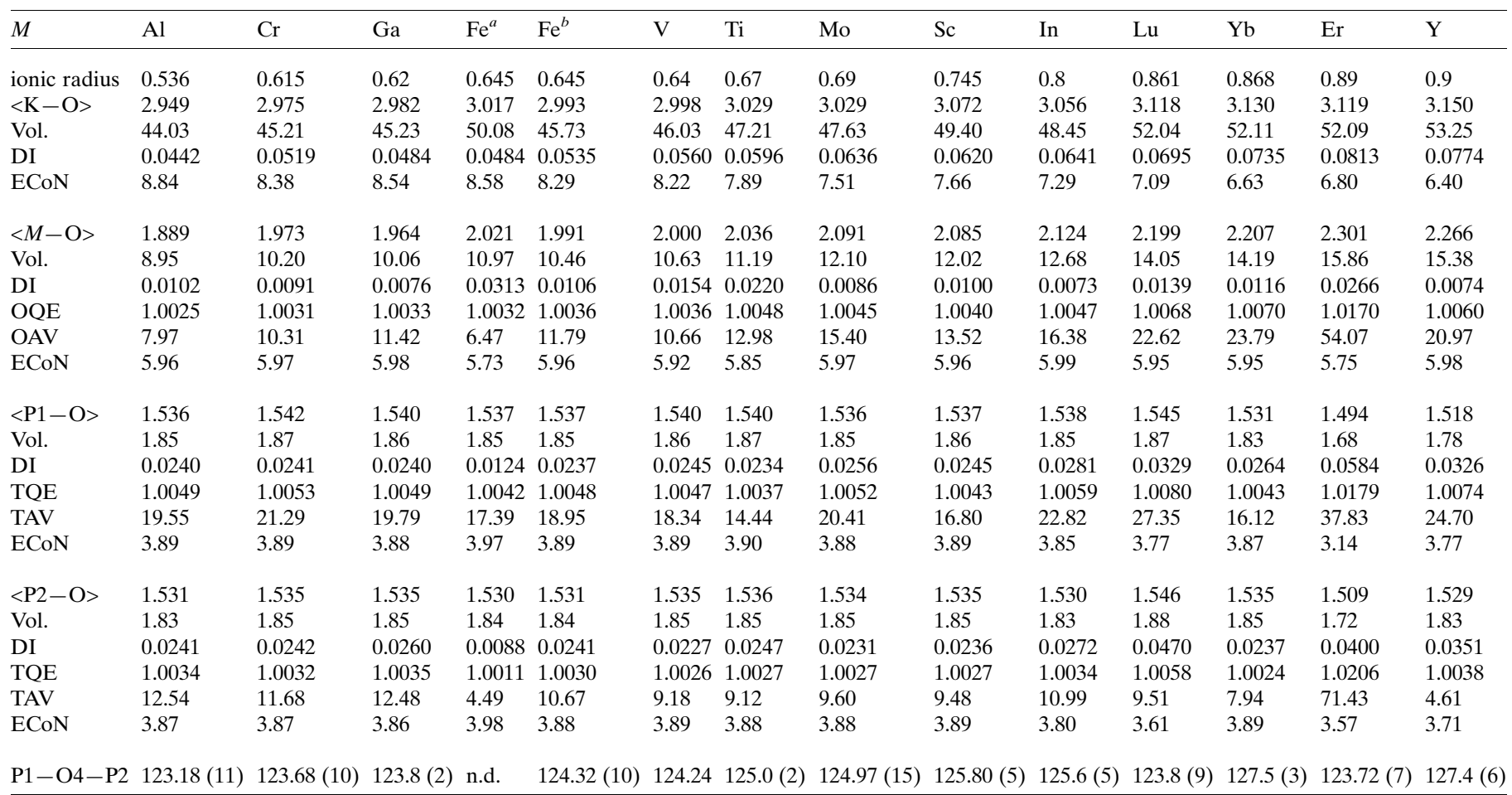

Notes: (a) data from Riou et al. (1988); (b) data from Genkin \& Timofeeva (1989).

$\mathrm{ScO}_{6}$ octahedron of one layer shares its $\mathrm{O}^{\mathrm{iii}}$ and $\mathrm{O}^{\mathrm{i}}$ corners (symmetry codes refer to Fig. 1) with one $\mathrm{PO}_{4}$ tetrahedron each of the layer below and above.

Generally, all corners of the octahedron are shared with neighbouring $\mathrm{PO}_{4}$ tetrahedra, whereby all oxygen atoms except $\mathrm{O} 4$ directly connect the octahedron with a pyrophosphate $\mathrm{P}_{2} \mathrm{O}_{7}$ group, and the $\mathrm{O}_{2}{ }^{\mathrm{ii}}$ and $\mathrm{O}^{\mathrm{iv}}$ oxygen atoms join the octahedron with two $\mathrm{PO}_{4}$ tetrahedra within the abovementioned layer parallel to (001). Additionally, the O1, $\mathrm{O}^{\text {iv }}$ and $\mathrm{O} 7$ oxygen atoms (Fig. 1) are also bonded to one $\mathrm{K}^{\mathrm{I}}$ cation each. The average $\mathrm{Sc}-\mathrm{O}$ bond length is $2.085 \AA$ while individual bond lengths range between $2.0736(7)$ and 2.1122 (6) A with one shorter bond ( $\mathrm{Sc}-\mathrm{O}^{\mathrm{i}}$ ) of 2.0346 (7) $\AA$. A similar behaviour with one significantly shorter $M-\mathrm{O}$ bond is also observed in other $\mathrm{K}^{\mathrm{III}} \mathrm{P}_{2} \mathrm{O}_{7}$ compounds and seems to be a more general feature. The $\mathrm{Sc}-\mathrm{O}^{\mathrm{i}}$ and the $\mathrm{Sc}-\mathrm{O}^{\mathrm{iii}}$ bonds, which point towards [001] and connect different (001) layers, both are the shortest within the $\mathrm{ScO}_{6}$ octahedron. Assuming that these two bonds are those to the axial oxygen atoms of the octahedron, the coordination polyhedron appears to be slightly compressed. Also, Ng \& Calvo (1973) noted for $\mathrm{KAlP}_{2} \mathrm{O}_{7}$ that the axial bonds are considerably shorter that the equatorial ones within the (001) layer and - more generally speaking - this is also found in other $\mathrm{KM}^{\mathrm{III}} \mathrm{P}_{2} \mathrm{O}_{7}$ compounds. The $\mathrm{ScO}_{6}$ octahedron in the title compound is only slightly distorted in terms of bond lengths and bond-angle variance (Table 1). It is worth noting that
$\mathrm{KAlP}_{2} \mathrm{O}_{7}$ shows the most regular octahedral coordination of all $\mathrm{KM}^{\mathrm{III}} \mathrm{P}_{2} \mathrm{O}_{7}$ structures compared here, and the distortion increases with increasing size of the octahedral cation as depicted in Fig. $3 a$. The average $\langle M-\mathrm{O}>$ bond lengths also scale well with the ionic radius of the $M$ site cation and are positively correlated (Fig. 3b).

Large heptagonal cavities are formed in the skeleton of octahedral and tetrahedral units that are made up from four tetrahedrally and two octahedrally coordinated sites within the (001) layer. The stacking of the layers leads to channels running parallel to [001] where the potassium cations are hosted. They are tenfold coordinated with $\mathrm{K}-\mathrm{O}$ bond lengths ranging between 2.7837 (7) $\AA$ and 3.3265 (9) $\AA$, the average $\mathrm{K}-\mathrm{O}$ bond length being $3.072 \AA$. As for $\langle M-\mathrm{O}\rangle$, the average $\mathrm{K}-\mathrm{O}$ bond length also increases with increasing size of the $M$ site cation, i.e. the channel size increases also.

Using bond-valence energy landscape map (BVEL) calculations, an estimation of possible diffusion pathways of alkali ions in a compound can be facilitated. Using the program SoftBV (Chen \& Adams, 2017; Chen et al., 2019) such calculations were performed on $\mathrm{KScP}_{2} \mathrm{O}_{7}$ and reveal two energy minima. The lowest lying minimum is indeed occupied by the $\mathrm{K}^{\mathrm{I}}$ cation, a second one is present at $x, y, z=0.271,0.317,0.438$ (interstitial $i 1$ ) and is unoccupied. A one-dimensional diffusion pathway is evident (Fig. 4), involving the $i 1$ position, and is oriented parallel to [001]. An estimated activation energy of $\sim 0.3 \mathrm{eV}$ would be needed to move a potassium ion from the 

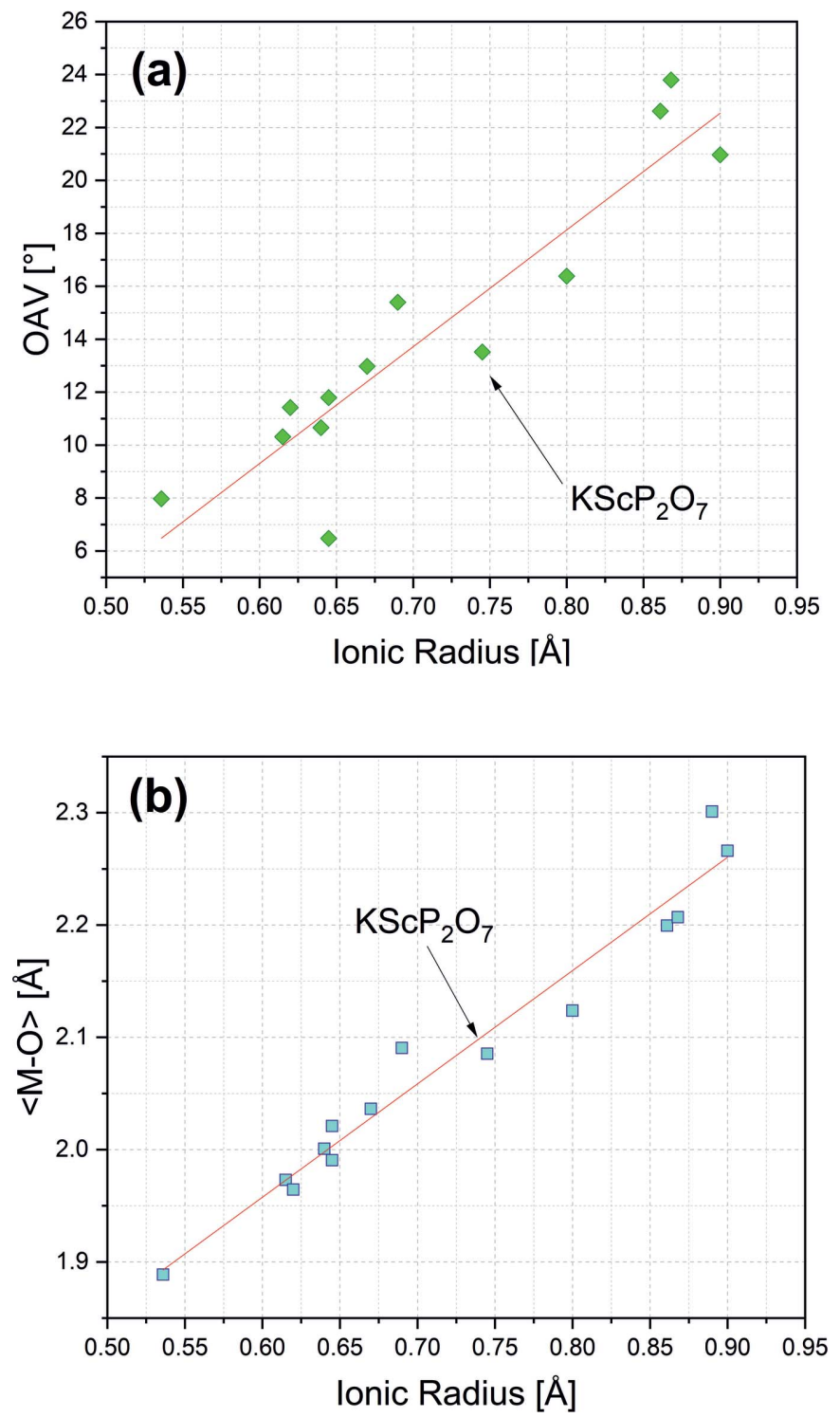

Figure 3

Variation of the octahedral angle variance (OAV) $(a)$ and the average $M^{\mathrm{III}}-\mathrm{O}$ bond lengths $(b)$ as a function of the ionic radius of the $M$ site cation across the series of $\mathrm{K}^{\mathrm{III}} \mathrm{P}_{2} \mathrm{O}_{7}$ compounds $(M=\mathrm{Al}$ to $\mathrm{Y})$.

regular $\mathrm{K}$ site to the interstitial $i 1$ site; to move it from $i 1$ to the next $\mathrm{K} 1$ site needs $\sim 1.3 \mathrm{eV}$. Interestingly, the percolation energy in e.g. $\mathrm{Fe}^{\mathrm{III}}, \mathrm{Mo}^{\mathrm{III}}$ and $\mathrm{In}^{\mathrm{III}}$ compounds of the $\mathrm{KM}^{\mathrm{III}} \mathrm{P}_{2} \mathrm{O}_{7}$ series is distinctly higher with around $1.8 \mathrm{eV}$ as estimated from BVEL maps. Generally, a partial substitution of trivalent cations by divalent ones might be of interest to increase the content of alkaline ions (here $\mathrm{K}^{\mathrm{I}}$ ), which most probably could be found on the interstitial $i 1$ site.

\section{Synthesis and crystallization}

The title compound was grown during attempts to synthesize NaSICON-type $\mathrm{K}_{3} \mathrm{Sc}_{2}\left(\mathrm{PO}_{4}\right)_{3}$ adopting a flux growth protocol set up by Sljukic et al. (1967). $\mathrm{Sc}_{2} \mathrm{O}_{3}$ and $\mathrm{KH}_{2} \mathrm{PO}_{4}$ were mixed in stoichiometric quantities (molar ratio 2:3) and $\mathrm{B}_{2} \mathrm{O}_{3}$ was added as a flux with a sixfold quantity of that of $\mathrm{Sc}_{2} \mathrm{O}_{3}$. The

Table 2

Experimental details.

Crystal data

Chemical formula

$M_{\text {r }}$

Crystal system, space group

$a, b, c(\AA)$

$\beta\left({ }^{\circ}\right)$

$V\left(\AA^{3}\right)$

Z

Radiation type

$\mu\left(\mathrm{mm}^{-1}\right)$

Crystal size (mm)

Data collection

Diffractometer

Absorption correction

$T_{\min }, T_{\max }$

No. of measured, independent and observed $[I>2 \sigma(I)]$ reflections

$R_{\text {int }}$

$(\sin \theta / \lambda)_{\max }\left(\AA^{-1}\right)$

Refinement

$R\left[F^{2}>2 \sigma\left(F^{2}\right)\right], w R\left(F^{2}\right), S$

No. of reflections

No. of parameters

$\Delta \rho_{\max }, \Delta \rho_{\min }\left(\mathrm{e} \AA^{-3}\right)$
Temperature (K)

\author{
$\mathrm{KScP}_{2} \mathrm{O}_{7}$ \\ 258 \\ Monoclinic, $P 2_{1} / c$ \\ 293 \\ 7.4634 (1), 10.3902 (1), 8.3747 (1) \\ 106.49 \\ $622.72(1)$ \\ 4 \\ Mo $K \alpha$ \\ 2.35 \\ $0.16 \times 0.09 \times 0.08$
}

Bruker SMART APEX CCD

Multi-scan (SADABS; Krause et al., 2015)

$0.38,0.52$

20981, 2985, 2850

0.021

0.837

Computer programs: APEX2 and SAINT (Bruker, 2012), SIR2014 (Burla et al., 2005), SHELXL2014/7 (Sheldrick, 2015), DIAMOND (Brandenburg, 2006), ORTEP for Windows and Win $G X$ publication routines (Farrugia, 2012).

complete mixture was transferred to a platinum crucible, covered with a lid, and heated in a chamber furnace to $1473 \mathrm{~K}$, held at this temperature for $24 \mathrm{~h}$ and then slowly cooled down to $1073 \mathrm{~K}$ at a rate of $3 \mathrm{~K} \mathrm{~h}^{-1}$. Between $1073 \mathrm{~K}$ and room temperature the cooling rate was $50 \mathrm{~K} \mathrm{~h}^{-1}$. The synthesis batch was immersed in hot water to dissolve the $\mathrm{B}_{2} \mathrm{O}_{3}$ and remaining $\mathrm{K}$-phosphates. The residual contained single-phase $\mathrm{KScP}_{2} \mathrm{O}_{7}$ as checked by powder $\mathrm{X}$-ray diffraction and showed

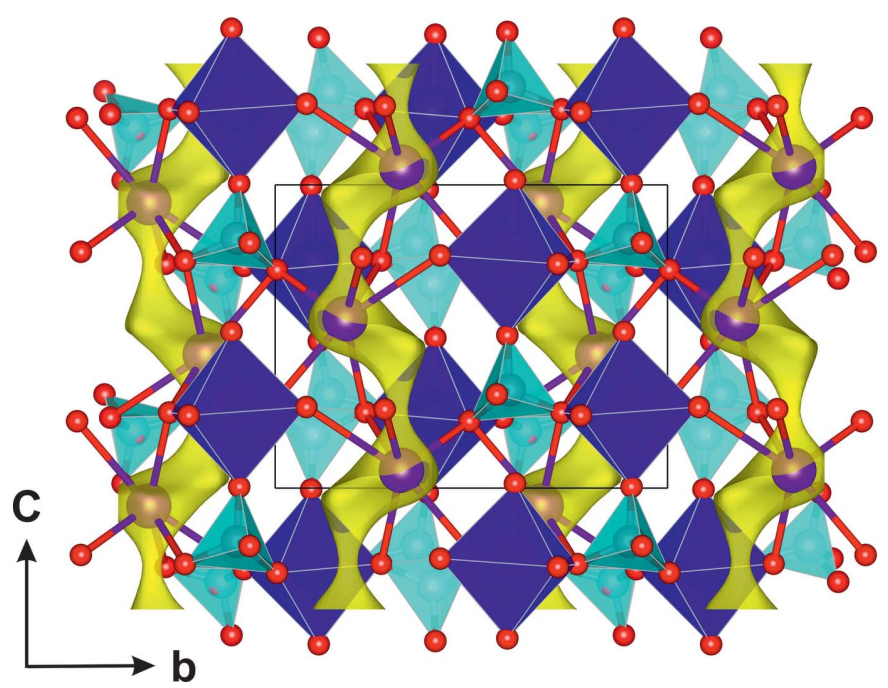

Figure 4

Bond-valence energy landscape map at levels of $1.5 \mathrm{eV}$ above the minimum (yellow) viewed along [100]; the crystal structure of $\mathrm{KScP}_{2} \mathrm{O}_{7}$ is overlayed, the $\mathrm{K}$ sites lie within channels. 
single crystals of irregular to needle-like form with welldeveloped faces. The crystals are colorless and highly transparent with sizes up to $140 \mu \mathrm{m}$ in lengths and $\sim 80 \mu \mathrm{m}$ in diameter.

\section{Refinement}

Crystal data, data collection and structure refinement details are summarized in Table 2.

\section{References}

Benhamada, L., Grandin, A., Borel, M. M., Leclaire, A. \& Raveau, B. (1991). Acta Cryst. C47, 424-425.

Brandenburg, K. (2006). DIAMOND. Crystal Impact GbR, Bonn, Germany.

Bruker (2012). APEX2 and SAINT. Bruker AXS Inc, Madison, Wisconsin, USA.

Burla, M. C., Caliandro, R., Camalli, M., Carrozzini, B., Cascarano, G. L., De Caro, L., Giacovazzo, C., Polidori, G. \& Spagna, R. (2005). J. Appl. Cryst. 38, 381-388.

Chaker, M., Horchani-Naifer, K. \& Férid, M. (2016). J. Alloys Compd. 688, 104-110.

Chen, H. \& Adams, S. (2017). IUCrJ, 4, 614-625.

Chen, H., Wong, L. L. \& Adams, S. (2019). Acta Cryst. B75, 18-33.

Chen, J. J., Wang, C. C. \& Lii, K. H. (1989). Acta Cryst. C45, 673-675.

Clearfield, A. (1988). Chem. Rev. 88, 125-148.

Farrugia, L. J. (2012). J. Appl. Cryst. 45, 849-854.

Genkin, E. A. \& Timofeeva, V. A. (1989). J. Struct. Chem. 30, 149151.

Gentil, S., Andreica, D., Lujan, M., Rivera, J. P., Kubel, F. \& Schmid, H. (1997). Ferroelectrics, 204, 35-44.

Hagerman, M. E. \& Poeppelmeier, K. R. (1995). Chem. Mater. 7, 602621.
Horchani-Naifer, K. \& Férid, M. (2007). Acta Cryst. E63, i33-i34.

Krause, L., Herbst-Irmer, R., Sheldrick, G. M. \& Stalke, D. (2015). J. Appl. Cryst. 48, 3-10.

Ladenstein, L., Lunghammer, S., Wang, E. Y., Miara, L. J., Wilkening, M. H. R., Redhammer, G. J. \& Rettenwander, D. (2020). J. Phys. Energ. 2, 035003.

Leclaire, A., Borel, M. M., Grandin, A. \& Raveau, B. (1989). J. Solid State Chem. 78, 220-226.

Momma, K. \& Izumi, F. (2011). J. Appl. Cryst. 44, 1272-1276.

Ng, H. N. \& Calvo, C. (1973). Can. J. Chem. 51, 2613-2620.

Redhammer, G. J., Tippelt, G., Stahl, Q., Benisek, A. \& Rettenwander, D. (2020). Acta Cryst. B76, submitted [Co-editor code RM5035].

Rettenwander, D., Redhammer, G. J., Guin, M., Benisek, A., Krüger, H., Guillon, O., Wilkening, M., Tietz, F. \& Fleig, J. (2018). Chem. Mater. 30, 1776-1781.

Riou, D., Labbe, P. \& Goreaud, M. (1988). Eur. J. Solid State Inorg. Chem. 25, 215-229.

Shannon, R. D. (1976). Acta Cryst. A32, 751-767.

Sheldrick, G. M. (2015). Acta Cryst. C71, 3-8.

Sljukic, M., Matkovic, B., Prodic, B. \& Scavnicar, S. (1967). Croat. Chem. Acta, 39, 145-.

Vìtiņš, G., Kaņepe, Z., Vîtiņš, A., Ronis, J., Dindūne, A. \& Lūsis, A. (2000). J. Solid State Electrochem. 4, 146-152.

Yuan, J. L., Zhang, H., Chen, H. H., Yang, X. X., Zhao, J. T. \& Gu, M. (2007). J. Solid State Chem. 180, 3381-3387.

Zagorac, D., Müller, H., Ruehl, S., Zagorac, J. \& Rehme, S. (2019). J. Appl. Cryst. 52, 918-925.

Zatovsky, I. V., Slobodyanik, N. S., Kowalski, A. \& Slyva, T. Yu. (2000). Dopov. Nats. Akad. Nauk Ukr. pp. 151-155.

Zhang, X. M., Zhang, H. Z., Chen, C., Kim, S. I. \& Seo, H. J. (2016). Mater. Lett. 168, 207-209.

Zhang, Y. C., Cheng, W. D., Wu, D. S., Zhang, H., Chen, D. G., Gong, Y. J. \& Kan, Z. G. (2004). Chem. Mater. 16, 4150-4159. 


\section{supporting information}

Acta Cryst. (2020). E76, 1412-1416 [https://doi.org/10.1107/S2056989020010427]

\section{The crystal structure of $\mathrm{KScP}_{2} \mathrm{O}_{7}$}

\section{Günther J. Redhammer and Gerold Tippelt}

\section{Computing details}

Data collection: APEX2 (Bruker, 2012); cell refinement: SAINT (Bruker, 2012); data reduction: SAINT (Bruker, 2012); program(s) used to solve structure: SIR2014 (Burla et al., 2005); program(s) used to refine structure: SHELXL2014/7 (Sheldrick, 2015); molecular graphics: DIAMOND (Brandenburg, 2006), ORTEP for Windows (Farrugia, 2012); software used to prepare material for publication: WinGX publication routines (Farrugia, 2012).

Potassium scandium diphosphate

Crystal data

$\mathrm{KScP}_{2} \mathrm{O}_{7}$

$M_{r}=258$

Monoclinic, $P 22_{1} / c$

Hall symbol: -P 2ybc

$a=7.4634(1) \AA$

$b=10.3902(1) \AA$

$c=8.3747(1) \AA$

$\beta=106.49^{\circ}$

$V=622.72(1) \AA^{3}$

$Z=4$

\section{Data collection}

Bruker SMART APEX CCD

diffractometer

Graphite monochromator rotation, $\omega$-scans at 4 different $\varphi$ positions

Absorption correction: multi-scan

(SADABS; Krause et al., 2015)

$T_{\min }=0.38, T_{\max }=0.52$

20981 measured reflections

\section{Refinement}

Refinement on $F^{2}$

Least-squares matrix: full

$R\left[F^{2}>2 \sigma\left(F^{2}\right)\right]=0.018$

$w R\left(F^{2}\right)=0.049$

$S=1.05$

2985 reflections

101 parameters

0 restraints

0 constraints

Primary atom site location: structure-invariant direct methods
$F(000)=504$

$D_{\mathrm{x}}=2.752 \mathrm{Mg} \mathrm{m}^{-3}$

Mo $K \alpha$ radiation, $\lambda=0.71073 \AA$

Cell parameters from 20981 reflections

$\theta=2.9-36.5^{\circ}$

$\mu=2.35 \mathrm{~mm}^{-1}$

$T=293 \mathrm{~K}$

Prismatic, colorless

$0.16 \times 0.09 \times 0.08 \mathrm{~mm}$

2985 independent reflections

2850 reflections with $I>2 \sigma(I)$

$R_{\text {int }}=0.021$

$\theta_{\text {max }}=36.5^{\circ}, \theta_{\min }=2.9^{\circ}$

$h=-12 \rightarrow 12$

$k=-17 \rightarrow 17$

$l=-13 \rightarrow 13$

Secondary atom site location: structureinvariant direct methods

$w=1 /\left[\sigma^{2}\left(F_{\mathrm{o}}{ }^{2}\right)+(0.0257 P)^{2}+0.274 P\right]$ where $P=\left(F_{\mathrm{o}}^{2}+2 F_{\mathrm{c}}{ }^{2}\right) / 3$

$(\Delta / \sigma)_{\max }=0.001$

$\Delta \rho_{\max }=0.79 \mathrm{e} \AA^{-3}$

$\Delta \rho_{\min }=-0.66$ e $\AA^{-3}$

Extinction correction: SHELXL2014/7

(Sheldrick 2015),

$\mathrm{Fc}^{*}=\mathrm{kFc}\left[1+0.001 \mathrm{xFc}^{2} \lambda^{3} / \sin (2 \theta)\right]^{-1 / 4}$

Extinction coefficient: 0.0429 (15) 


\section{Special details}

Geometry. All esds (except the esd in the dihedral angle between two 1.s. planes) are estimated using the full covariance matrix. The cell esds are taken into account individually in the estimation of esds in distances, angles and torsion angles; correlations between esds in cell parameters are only used when they are defined by crystal symmetry. An approximate (isotropic) treatment of cell esds is used for estimating esds involving l.s. planes.

Fractional atomic coordinates and isotropic or equivalent isotropic displacement parameters $\left(\hat{A}^{2}\right)$

\begin{tabular}{lllll}
\hline & $x$ & $y$ & $z$ & $U_{\text {iso }} * / U_{\text {eq }}$ \\
\hline K1 & $0.82123(3)$ & $0.67829(3)$ & $0.94124(3)$ & $0.02342(6)$ \\
Sc1 & $0.76534(2)$ & $0.09952(2)$ & $0.74255(2)$ & $0.00607(4)$ \\
P1 & $0.55749(3)$ & $0.36164(2)$ & $0.80923(2)$ & $0.00673(5)$ \\
P2 & $0.86703(3)$ & $0.40292(2)$ & $0.67637(3)$ & $0.00681(5)$ \\
O1 & $0.54575(9)$ & $0.22058(6)$ & $0.76034(9)$ & $0.01132(11)$ \\
O2 & $0.36433(9)$ & $0.42041(6)$ & $0.76741(9)$ & $0.01313(11)$ \\
O3 & $0.67337(11)$ & $0.38889(7)$ & $0.98492(9)$ & $0.01712(13)$ \\
O4 & $0.66159(9)$ & $0.43502(6)$ & $0.69104(8)$ & $0.01167(11)$ \\
O5 & $0.99440(9)$ & $0.50481(6)$ & $0.77947(8)$ & $0.01003(10)$ \\
O6 & $0.85616(11)$ & $0.40831(7)$ & $0.49554(8)$ & $0.01632(13)$ \\
O7 & $0.92157(9)$ & $0.27011(6)$ & $0.75056(8)$ & $0.01088(10)$ \\
\hline
\end{tabular}

Atomic displacement parameters $\left(\AA^{2}\right)$

\begin{tabular}{lllllll}
\hline & $U^{11}$ & $U^{22}$ & $U^{33}$ & $U^{12}$ & $U^{13}$ & $U^{23}$ \\
\hline K1 & $0.02013(10)$ & $0.02973(12)$ & $0.01843(10)$ & $0.00408(8)$ & $0.00226(7)$ & $-0.00089(8)$ \\
Sc1 & $0.00647(6)$ & $0.00539(6)$ & $0.00639(6)$ & $0.00013(4)$ & $0.00190(4)$ & $-0.00015(4)$ \\
P1 & $0.00662(8)$ & $0.00633(8)$ & $0.00731(8)$ & $0.00074(6)$ & $0.00208(6)$ & $-0.00046(6)$ \\
P2 & $0.00755(8)$ & $0.00703(8)$ & $0.00616(8)$ & $-0.00186(6)$ & $0.00246(6)$ & $-0.00017(5)$ \\
O1 & $0.0091(2)$ & $0.0070(2)$ & $0.0185(3)$ & $0.00026(18)$ & $0.0050(2)$ & $-0.00208(19)$ \\
O2 & $0.0086(2)$ & $0.0100(2)$ & $0.0214(3)$ & $0.00284(19)$ & $0.0052(2)$ & $-0.0002(2)$ \\
O3 & $0.0209(3)$ & $0.0207(3)$ & $0.0073(3)$ & $0.0010(3)$ & $-0.0001(2)$ & $-0.0025(2)$ \\
O4 & $0.0086(2)$ & $0.0124(2)$ & $0.0150(3)$ & $0.00151(19)$ & $0.0050(2)$ & $0.0054(2)$ \\
O5 & $0.0103(2)$ & $0.0097(2)$ & $0.0107(2)$ & $-0.00398(18)$ & $0.00393(19)$ & $-0.00307(18)$ \\
O6 & $0.0199(3)$ & $0.0229(3)$ & $0.0069(2)$ & $-0.0082(2)$ & $0.0049(2)$ & $-0.0016(2)$ \\
O7 & $0.0098(2)$ & $0.0069(2)$ & $0.0159(3)$ & $-0.00059(18)$ & $0.0035(2)$ & $0.00091(19)$ \\
& & & & & &
\end{tabular}

Geometric parameters $\left(\AA,{ }^{\circ}\right)$

\begin{tabular}{llll}
\hline $\mathrm{K} 1-\mathrm{O} 5$ & $2.7837(7)$ & $\mathrm{P} 1-\mathrm{O} 3$ & $1.5068(7)$ \\
$\mathrm{K} 1-\mathrm{O} 7^{\mathrm{i}}$ & $2.7966(7)$ & $\mathrm{P} 1-\mathrm{O} 2$ & $1.5123(7)$ \\
$\mathrm{K} 1-\mathrm{O} 1^{\mathrm{ii}}$ & $2.8141(7)$ & $\mathrm{P} 1-\mathrm{O} 1$ & $1.5176(6)$ \\
$\mathrm{K} 1-\mathrm{O} 7^{\mathrm{iii}}$ & $2.9876(7)$ & $\mathrm{P} 1-\mathrm{O} 4$ & $3.5569(3)$ \\
$\mathrm{K} 1-\mathrm{O} 5^{\mathrm{i}}$ & $3.0259(7)$ & $\mathrm{P} 1-\mathrm{K} 1^{\mathrm{vii}}(6)$ \\
$\mathrm{K} 1-\mathrm{O} 2^{\mathrm{ii}}$ & $3.1505(7)$ & $\mathrm{P} 2-\mathrm{O} 6$ & $1.4944(7)$ \\
$\mathrm{K} 1-\mathrm{O} 3$ & $3.2592(8)$ & $\mathrm{P} 2-\mathrm{O} 5$ & $1.5178(6)$ \\
$\mathrm{K} 1-\mathrm{O} 4$ & $3.2835(7)$ & $\mathrm{P} 2-\mathrm{O} 7$ & $1.5207(6)$ \\
$\mathrm{K} 1-\mathrm{O} 2^{\mathrm{iv}}$ & $3.2927(7)$ & $\mathrm{P} 2-\mathrm{O} 4$ & $1.6076(6)$ \\
$\mathrm{K} 1-\mathrm{O} 6^{\mathrm{ii}}$ & $3.3265(9)$ & $\mathrm{P} 2-\mathrm{K} 1^{\mathrm{i}}$ & $3.4856(3)$
\end{tabular}




\begin{tabular}{|c|c|c|c|}
\hline $\mathrm{K} 1-\mathrm{P} 2^{\mathrm{i}}$ & $3.4856(3)$ & $\mathrm{P} 2-\mathrm{K} 1^{\text {viii }}$ & $3.6237(3)$ \\
\hline $\mathrm{K} 1-\mathrm{P} 1^{\mathrm{ii}}$ & $3.5570(3)$ & $\mathrm{O} 1-\mathrm{K} 1^{\mathrm{vii}}$ & $2.8141(7)$ \\
\hline $\mathrm{Sc} 1-\mathrm{O}^{\mathrm{v}}$ & $2.0346(7)$ & $\mathrm{O} 2-\mathrm{Sc}^{\mathrm{ii}}$ & $2.0883(6)$ \\
\hline $\mathrm{Sc} 1-\mathrm{O} 3^{\mathrm{vi}}$ & $2.0736(7)$ & $\mathrm{O} 2-\mathrm{K} 1^{\mathrm{vii}}$ & $3.1505(7)$ \\
\hline $\mathrm{Sc} 1-\mathrm{O} 2^{\mathrm{vii}}$ & $2.0884(6)$ & $\mathrm{O} 2-\mathrm{K} 1^{\text {iv }}$ & $3.2927(7)$ \\
\hline $\mathrm{Sc} 1-\mathrm{O} 5^{\mathrm{viii}}$ & $2.0989(6)$ & $\mathrm{O} 3-\mathrm{Sc}^{\mathrm{v}}$ & $2.0736(7)$ \\
\hline $\mathrm{Sc} 1-\mathrm{O} 1$ & $2.1045(6)$ & $\mathrm{O} 5-\mathrm{Sc} 1^{\mathrm{iii}}$ & $2.0990(6)$ \\
\hline $\mathrm{Sc} 1-\mathrm{O} 7$ & $2.1122(6)$ & $\mathrm{O} 5-\mathrm{K} 1^{\mathrm{i}}$ & $3.0259(7)$ \\
\hline $\mathrm{Sc} 1-\mathrm{K} 1^{\mathrm{viii}}$ & 3.9059 (3) & $\mathrm{O} 6-\mathrm{Sc}^{\mathrm{vi}}$ & $2.0346(7)$ \\
\hline $\mathrm{Sc} 1-\mathrm{K} 1^{\mathrm{vi}}$ & $3.9333(3)$ & $\mathrm{O} 6-\mathrm{K} 1^{\mathrm{viii}}$ & $3.3265(9)$ \\
\hline $\mathrm{Sc} 1-\mathrm{K} 1^{\mathrm{i}}$ & $4.1501(3)$ & $\mathrm{O} 7-\mathrm{K} 1^{\mathrm{i}}$ & $2.7966(7)$ \\
\hline $\mathrm{Sc} 1-\mathrm{K} 1^{\mathrm{vii}}$ & $4.2870(3)$ & $\mathrm{O} 7-\mathrm{K} 1^{\text {viii }}$ & $2.9875(7)$ \\
\hline $\mathrm{O} 5-\mathrm{K} 1-\mathrm{O}^{\mathrm{i}}$ & $106.35(2)$ & $\mathrm{O} 2^{\mathrm{vii}}-\mathrm{Sc} 1-\mathrm{K} 1^{\mathrm{vi}}$ & $56.82(2)$ \\
\hline $\mathrm{O} 5-\mathrm{K} 1-\mathrm{O} 1^{\mathrm{ii}}$ & $108.45(2)$ & $\mathrm{O}^{\mathrm{viii}}-\mathrm{Sc} 1-\mathrm{K} 1^{\mathrm{vi}}$ & $49.513(18)$ \\
\hline $\mathrm{O} 7^{\mathrm{i}}-\mathrm{K} 1-\mathrm{O} 1^{\mathrm{ii}}$ & $145.07(2)$ & $\mathrm{O} 1-\mathrm{Sc} 1-\mathrm{K} 1^{\mathrm{vi}}$ & $135.284(19)$ \\
\hline $\mathrm{O} 5-\mathrm{K} 1-\mathrm{O} 7^{\mathrm{iii}}$ & $59.145(18)$ & $\mathrm{O} 7-\mathrm{Sc} 1-\mathrm{K} 1^{\mathrm{vi}}$ & $118.557(19)$ \\
\hline $\mathrm{O} 7^{\mathrm{i}}-\mathrm{K} 1-\mathrm{O} 7^{\mathrm{iii}}$ & $93.300(18)$ & $\mathrm{K} 1^{\mathrm{viii}}-\mathrm{Sc} 1-\mathrm{K} 1^{\mathrm{vi}}$ & $70.210(7)$ \\
\hline $\mathrm{O} 1^{\mathrm{ii}-\mathrm{K}} 1-\mathrm{O} 7^{\mathrm{iii}}$ & $106.983(19)$ & $\mathrm{O} 6^{\mathrm{v}}-\mathrm{Sc} 1-\mathrm{K} 1^{\mathrm{i}}$ & $52.43(2)$ \\
\hline $\mathrm{O} 5-\mathrm{K} 1-\mathrm{O}^{5}$ & $78.29(2)$ & $\mathrm{O} 3^{\mathrm{vi}}-\mathrm{Sc} 1-\mathrm{K} 1^{\mathrm{i}}$ & $126.74(2)$ \\
\hline $\mathrm{O} 7^{\mathrm{i}}-\mathrm{K} 1-\mathrm{O} 5^{\mathrm{i}}$ & $50.565(17)$ & $\mathrm{O} 2^{\mathrm{vii}}-\mathrm{Sc} 1-\mathrm{K} 1^{\mathrm{i}}$ & $140.66(2)$ \\
\hline $\mathrm{O} 1^{\mathrm{ii}}-\mathrm{K} 1-\mathrm{O} 5^{\mathrm{i}}$ & $135.51(2)$ & 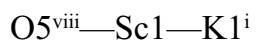 & $79.508(19)$ \\
\hline 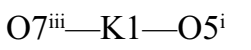 & $113.314(18)$ & $\mathrm{O} 1-\mathrm{Sc} 1-\mathrm{K} 1^{\mathrm{i}}$ & $94.313(18)$ \\
\hline $\mathrm{O} 5-\mathrm{K} 1-\mathrm{O} 2^{\mathrm{ii}}$ & $116.03(2)$ & $\mathrm{O} 7-\mathrm{Sc} 1-\mathrm{K} 1^{\mathrm{i}}$ & $37.741(18)$ \\
\hline $\mathrm{O} 7^{\mathrm{i}}-\mathrm{K} 1-\mathrm{O} 2^{\mathrm{ii}}$ & $115.93(2)$ & 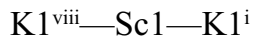 & $66.889(5)$ \\
\hline $\mathrm{O} 1^{\mathrm{ii}-\mathrm{K}} 1-\mathrm{O} 2^{\mathrm{ii}}$ & $48.919(17)$ & 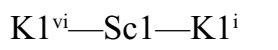 & $128.501(4)$ \\
\hline 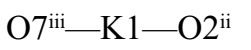 & $72.254(18)$ & $\mathrm{O} 6^{\mathrm{v}}-\mathrm{Sc} 1-\mathrm{K} 1^{\mathrm{vii}}$ & $112.70(2)$ \\
\hline $\mathrm{O} 5^{\mathrm{i}}-\mathrm{K} 1-\mathrm{O} 2^{\mathrm{ii}}$ & 164.277 (19) & $\mathrm{O} 3^{\mathrm{vi}}-\mathrm{Sc} 1-\mathrm{K} 1^{\mathrm{vii}}$ & $67.21(2)$ \\
\hline $\mathrm{O} 5-\mathrm{K} 1-\mathrm{O} 3$ & $71.10(2)$ & $\mathrm{O} 2^{\mathrm{vii}}-\mathrm{Sc} 1-\mathrm{K} 1^{\mathrm{vii}}$ & $75.012(19)$ \\
\hline $\mathrm{O} 7^{\mathrm{i}}-\mathrm{K} 1-\mathrm{O} 3$ & $103.820(19)$ & $\mathrm{O} 5^{\mathrm{viii}}-\mathrm{Sc} 1-\mathrm{K} 1^{\mathrm{vii}}$ & $150.317(19)$ \\
\hline $\mathrm{O} 1 \mathrm{ii}-\mathrm{K} 1-\mathrm{O} 3$ & 84.749 (19) & $\mathrm{O} 1-\mathrm{Sc} 1-\mathrm{K} 1^{\mathrm{vii}}$ & $34.402(18)$ \\
\hline $\mathrm{O} 7{ }^{\mathrm{iii}}-\mathrm{K} 1-\mathrm{O} 3$ & $130.140(19)$ & $\mathrm{O} 7-\mathrm{Sc} 1-\mathrm{K} 1^{\mathrm{vii}}$ & $110.455(18)$ \\
\hline $\mathrm{O} 5-\mathrm{i}-\mathrm{K} 1-\mathrm{O} 3$ & $55.064(17)$ & $\mathrm{K} 1^{\mathrm{viii}}-\mathrm{Sc} 1-\mathrm{K} 1^{\mathrm{vii}}$ & $131.220(7)$ \\
\hline $\mathrm{O} 22^{\mathrm{ii}}-\mathrm{K} 1-\mathrm{O} 3$ & $133.582(19)$ & $\mathrm{K} 1^{\mathrm{vi}}-\mathrm{Sc} 1-\mathrm{K} 1^{\mathrm{vii}}$ & $101.144(6)$ \\
\hline $\mathrm{O} 5-\mathrm{K} 1-\mathrm{O} 4$ & $47.582(17)$ & $\mathrm{K} 1^{\mathrm{i}}-\mathrm{Sc} 1-\mathrm{K} 1^{\mathrm{vii}}$ & $128.415(5)$ \\
\hline $\mathrm{O} 7^{\mathrm{i}}-\mathrm{K} 1-\mathrm{O} 4$ & 140.014 (19) & $\mathrm{O} 3-\mathrm{P} 1-\mathrm{O} 2$ & $113.29(4)$ \\
\hline $\mathrm{O} 1 \mathrm{ii}-\mathrm{K} 1-\mathrm{O} 4$ & $67.811(18)$ & $\mathrm{O} 3-\mathrm{P} 1-\mathrm{O} 1$ & $114.72(4)$ \\
\hline $\mathrm{O} 7^{\mathrm{iii}}-\mathrm{K} 1-\mathrm{O} 4$ & $94.293(17)$ & $\mathrm{O} 2-\mathrm{P} 1-\mathrm{O} 1$ & $110.44(4)$ \\
\hline 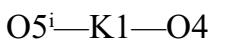 & $90.703(17)$ & $\mathrm{O} 3-\mathrm{P} 1-\mathrm{O} 4$ & $105.51(4)$ \\
\hline $\mathrm{O} 2^{\mathrm{ii}}-\mathrm{K} 1-\mathrm{O} 4$ & $103.768(18)$ & $\mathrm{O} 2-\mathrm{P} 1-\mathrm{O} 4$ & $105.11(4)$ \\
\hline $\mathrm{O} 3-\mathrm{K} 1-\mathrm{O} 4$ & $44.629(17)$ & $\mathrm{O} 1-\mathrm{P} 1-\mathrm{O} 4$ & $106.97(4)$ \\
\hline $\mathrm{O} 5-\mathrm{K} 1-\mathrm{O} 2^{\mathrm{iv}}$ & 120.669 (19) & $\mathrm{O} 3-\mathrm{P} 1-\mathrm{K} 1^{\mathrm{vii}}$ & $144.71(3)$ \\
\hline $\mathrm{O} 7^{\mathrm{i}}-\mathrm{K} 1-\mathrm{O} 2^{\mathrm{iv}}$ & $72.463(19)$ & $\mathrm{O} 2-\mathrm{P} 1-\mathrm{K} 1^{\mathrm{vii}}$ & $62.23(3)$ \\
\hline $\mathrm{O} 1^{\mathrm{ii}-}-\mathrm{K} 1-\mathrm{O} 2^{\mathrm{iv}}$ & $87.172(19)$ & $\mathrm{O} 1-\mathrm{P} 1-\mathrm{K} 1^{\mathrm{vii}}$ & $49.33(3)$ \\
\hline $\mathrm{O} 7^{\mathrm{iii}}-\mathrm{K} 1-\mathrm{O} 2^{\mathrm{iv}}$ & 165.347 (19) & $\mathrm{O} 4-\mathrm{P} 1-\mathrm{K} 1^{\mathrm{vii}}$ & $109.38(3)$ \\
\hline $\mathrm{O} 5^{\mathrm{i}}-\mathrm{K} 1-\mathrm{O} 2^{\mathrm{iv}}$ & $54.972(17)$ & $\mathrm{O} 3-\mathrm{P} 1-\mathrm{K} 1$ & $56.74(3)$ \\
\hline $\mathrm{O} 2^{\mathrm{ii}}-\mathrm{K} 1-\mathrm{O} 2^{\mathrm{iv}}$ & $116.65(2)$ & $\mathrm{O} 2-\mathrm{P} 1-\mathrm{K} 1$ & $95.62(3)$ \\
\hline $\mathrm{O} 3-\mathrm{K} 1-\mathrm{O} 2^{\mathrm{iv}}$ & $53.305(18)$ & $\mathrm{O} 1-\mathrm{P} 1-\mathrm{K} 1$ & $153.20(3)$ \\
\hline
\end{tabular}




$$
\begin{aligned}
& \mathrm{O} 4-\mathrm{K} 1-\mathrm{O} 2^{\text {iv }} \\
& \mathrm{O} 5-\mathrm{K} 1-\mathrm{O}^{\mathrm{iii}} \\
& \mathrm{O} 7^{\mathrm{i}}-\mathrm{K} 1-\mathrm{O}^{\mathrm{iii}} \\
& \mathrm{O} 1^{\mathrm{ii}}-\mathrm{K} 1-\mathrm{O} 6^{\mathrm{iii}}
\end{aligned}
$$

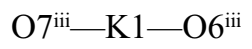

$$
\begin{aligned}
& \mathrm{O} 5^{\mathrm{i}}-\mathrm{K} 1-\mathrm{O}^{\mathrm{iii}}
\end{aligned}
$$

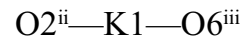

$$
\begin{aligned}
& \mathrm{O} 3-\mathrm{K} 1-\mathrm{O}^{\mathrm{iii}} \\
& \mathrm{O} 4-\mathrm{K} 1-\mathrm{O} 6^{\mathrm{iii}} \\
& \mathrm{O} 2^{\mathrm{iv}}-\mathrm{K} 1-\mathrm{O} 6^{\mathrm{iii}} \\
& \mathrm{O} 5-\mathrm{K} 1-\mathrm{P} 2^{\mathrm{i}} \\
& \mathrm{O} 7^{\mathrm{i}}-\mathrm{K} 1-\mathrm{P} 2^{\mathrm{i}}
\end{aligned}
$$

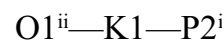

$$
\begin{aligned}
& \mathrm{O} 7^{\mathrm{iii}}-\mathrm{K} 1-\mathrm{P} 2^{\mathrm{i}} \\
& \mathrm{O} 5^{\mathrm{i}}-\mathrm{K} 1-\mathrm{P} 2^{\mathrm{i}} \\
& \mathrm{O} 2^{\mathrm{ii}}-\mathrm{K} 1-\mathrm{P} 2^{\mathrm{i}} \\
& \mathrm{O} 3-\mathrm{K} 1-\mathrm{P} 2^{\mathrm{i}} \\
& \mathrm{O} 4-\mathrm{K} 1-\mathrm{P} 2^{\mathrm{i}} \\
& \mathrm{O} 2^{\mathrm{iv}}-\mathrm{K} 1-\mathrm{P} 2^{\mathrm{i}} \\
& \mathrm{O} 6^{\mathrm{iii}}-\mathrm{K} 1-\mathrm{P} 2^{\mathrm{i}} \\
& \mathrm{O} 5-\mathrm{K} 1-\mathrm{P} 1^{\mathrm{ii}} \\
& \mathrm{O} 7^{\mathrm{i}}-\mathrm{K} 1-\mathrm{P} 1^{\mathrm{ii}} \\
& \mathrm{O} 1^{\mathrm{ii}}-\mathrm{K} 1-\mathrm{P} 1^{\mathrm{ii}}
\end{aligned}
$$

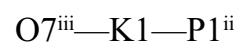

$$
\begin{aligned}
& \mathrm{O} 5^{\mathrm{i}}-\mathrm{K} 1-\mathrm{P} 1^{\mathrm{ii}} \\
& \mathrm{O} 2^{\mathrm{ii}-\mathrm{K}} 1-\mathrm{P} 1^{\mathrm{ii}} \\
& \mathrm{O} 3-\mathrm{K} 1-\mathrm{P} 1^{\mathrm{ii}} \\
& \mathrm{O} 4-\mathrm{K} 1-\mathrm{P} 1^{\mathrm{ii}} \\
& \mathrm{O} 2^{\mathrm{iv}}-\mathrm{K} 1-\mathrm{P} 1^{\mathrm{ii}} \\
& \mathrm{O}^{\mathrm{iii}}-\mathrm{K} 1-\mathrm{P} 1^{\mathrm{ii}} \\
& \mathrm{P} 2^{\mathrm{i}}-\mathrm{K} 1-\mathrm{P} 1^{\mathrm{ii}} \\
& \mathrm{O}^{\mathrm{v}}-\mathrm{Sc} 1-\mathrm{O}^{\mathrm{vi}} \\
& \mathrm{O}^{\mathrm{v}}-\mathrm{Sc} 1-\mathrm{O} 2^{\mathrm{vii}} \\
& \mathrm{O}^{\mathrm{vi}}-\mathrm{Sc} 1-\mathrm{O}^{\text {vii }} \\
& \mathrm{O}^{\mathrm{v}}-\mathrm{Sc} 1-\mathrm{O} 5^{\mathrm{viii}} \\
& \mathrm{O}^{\mathrm{vi}}-\mathrm{Sc} 1-\mathrm{O}^{\text {viii }}
\end{aligned}
$$

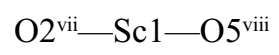

$$
\begin{aligned}
& \mathrm{O}^{\mathrm{v}}-\mathrm{Sc} 1-\mathrm{O} 1
\end{aligned}
$$

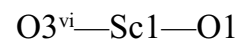

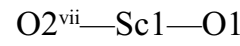

$$
\begin{aligned}
& \text { O5 }{ }^{\text {viii- }} \mathrm{Sc} 1-\mathrm{O} 1 \\
& \mathrm{O}^{\mathrm{v}}-\mathrm{Sc} 1-\mathrm{O} 7 \\
& \mathrm{O}^{\text {vi }}-\mathrm{Sc} 1-\mathrm{O} 7 \\
& \mathrm{O} 2^{\mathrm{vii}}-\mathrm{Sc} 1-\mathrm{O} 7 \\
& \text { O5 }{ }^{\text {vii }-S c 1-O 7 ~} \\
& \mathrm{O} 1-\mathrm{Sc} 1-\mathrm{O} 7 \\
& \mathrm{O}^{\mathrm{v}}-\mathrm{Sc} 1-\mathrm{K} 1^{\mathrm{viii}} \\
& \mathrm{O}^{\text {vi }}-\mathrm{Sc} 1-\mathrm{K} 1^{\text {viii }}
\end{aligned}
$$

$94.610(18)$

97.331 (19)

55.891 (18)

121.365 (19)

46.342 (17)

100.309 (18)

72.478 (18)

153.88 (2)

$140.332(18)$

$122.924(18)$

90.537 (15)

$25.078(13)$

150.579 (16)

102.011 (14)

25.736 (12)

140.943 (16)

80.358 (14)

115.250 (14)

$63.593(13)$

76.323 (13)

117.439 (16)

$131.596(16)$

24.144 (13)

$92.076(14)$

154.610 (15)

25.133 (12)

108.821 (15)

$87.268(13)$

$99.950(13)$

97.304 (14)

151.985 (9)

$178.96(3)$

$91.12(3)$

89.85 (3)

$91.86(3)$

88.54 (3)

88.65 (3)

$89.20(3)$

$90.26(3)$

100.03 (3)

171.24 (3)

89.01 (3)

$90.06(3)$

173.98 (3)

85.32 (2)

$86.00(2)$

110.60 (2)

69.08 (2)
$\mathrm{O} 4-\mathrm{P} 1-\mathrm{K} 1$

$\mathrm{K} 1{ }^{\mathrm{vii}}-\mathrm{P} 1-\mathrm{K} 1$

$\mathrm{O} 6-\mathrm{P} 2-\mathrm{O} 5$

O6-P2-O7

$\mathrm{O} 5-\mathrm{P} 2-\mathrm{O} 7$

$\mathrm{O} 6-\mathrm{P} 2-\mathrm{O} 4$

$\mathrm{O} 5-\mathrm{P} 2-\mathrm{O} 4$

$\mathrm{O} 7-\mathrm{P} 2-\mathrm{O} 4$

$\mathrm{O} 6-\mathrm{P} 2-\mathrm{K} 1^{\mathrm{i}}$

$\mathrm{O} 5-\mathrm{P} 2-\mathrm{K} 1^{\mathrm{i}}$

$\mathrm{O} 7-\mathrm{P} 2-\mathrm{K} 1^{\mathrm{i}}$

$\mathrm{O} 4-\mathrm{P} 2-\mathrm{K} 1^{\mathrm{i}}$

$\mathrm{O} 6-\mathrm{P} 2-\mathrm{K} 1^{\text {viii }}$

$\mathrm{O} 5-\mathrm{P} 2-\mathrm{K} 1^{\text {viii }}$

O7-P2-K1 ${ }^{\text {viii }}$

$\mathrm{O} 4-\mathrm{P} 2-\mathrm{K} 1^{\text {viii }}$

$\mathrm{K} 1{ }^{\mathrm{i}}-\mathrm{P} 2-\mathrm{K} 1^{\text {viii }}$

$\mathrm{O} 6-\mathrm{P} 2-\mathrm{K} 1$

$\mathrm{O} 5-\mathrm{P} 2-\mathrm{K} 1$

O7-P2-K1

$\mathrm{O} 4-\mathrm{P} 2-\mathrm{K} 1$

$\mathrm{K} 1{ }^{\mathrm{i}}-\mathrm{P} 2-\mathrm{K} 1$

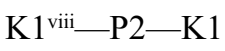

$\mathrm{P} 1-\mathrm{O} 1-\mathrm{Sc} 1$

$\mathrm{P} 1-\mathrm{O} 1-\mathrm{K} 1^{\text {vii }}$

$\mathrm{Sc} 1-\mathrm{O} 1-\mathrm{K} 1^{\text {vii }}$

$\mathrm{P} 1-\mathrm{O} 2-\mathrm{Sc}^{\mathrm{ii}}$

$\mathrm{P} 1-\mathrm{O} 2-\mathrm{K} 1^{\text {vii }}$

$\mathrm{Sc} 1^{\mathrm{ii}}-\mathrm{O} 2-\mathrm{K} 1^{\mathrm{vii}}$

$\mathrm{P} 1-\mathrm{O} 2-\mathrm{K} 1^{\mathrm{iv}}$

$\mathrm{Sc} 1^{\mathrm{ii}}-\mathrm{O} 2-\mathrm{K} 1^{\mathrm{iv}}$

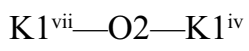

$\mathrm{P} 1-\mathrm{O} 3-\mathrm{Sc}^{\mathrm{v}}$

$\mathrm{P} 1-\mathrm{O} 3-\mathrm{K} 1$

$\mathrm{Sc} 1^{\mathrm{v}}-\mathrm{O} 3-\mathrm{K} 1$

$\mathrm{P} 2-\mathrm{O} 4-\mathrm{P} 1$

$\mathrm{P} 2-\mathrm{O} 4-\mathrm{K} 1$

$\mathrm{P} 1-\mathrm{O} 4-\mathrm{K} 1$

$\mathrm{P} 2-\mathrm{O} 5-\mathrm{Sc} 1^{\mathrm{iii}}$

$\mathrm{P} 2-\mathrm{O} 5-\mathrm{K} 1$

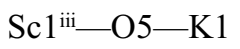

$\mathrm{P} 2-\mathrm{O} 5-\mathrm{K} 1^{\mathrm{i}}$

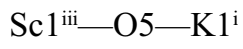

$\mathrm{K} 1-\mathrm{O} 5-\mathrm{K} 1^{\mathrm{i}}$

$\mathrm{P} 2-\mathrm{O} 6-\mathrm{Sc}^{\mathrm{vi}}$

$\mathrm{P} 2-\mathrm{O} 6-\mathrm{K} 1^{\text {viii }}$

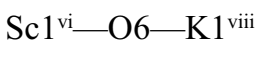

$\mathrm{P} 2-\mathrm{O} 7-\mathrm{Sc} 1$
$58.24(3)$

$152.503(8)$

113.29 (4)

112.29 (4)

110.41 (4)

106.93 (4)

105.59 (4)

107.91 (3)

140.37 (3)

$59.96(3)$

$51.21(3)$

112.48 (3)

66.61 (3)

104.90 (3)

53.74 (3)

148.68 (3)

77.363 (6)

126.27 (3)

42.96 (2)

121.13 (3)

$62.66(3)$

$77.719(8)$

146.911 (7)

127.58 (4)

106.53 (3)

120.60 (3)

140.31 (4)

92.64 (3)

124.31 (3)

106.05 (3)

91.12 (2)

87.202 (17)

162.86 (5)

100.52 (4)

92.33 (3)

125.80 (4)

$91.56(3)$

97.07 (3)

133.60 (4)

115.23 (3)

105.40 (2)

94.31 (3)

98.65 (2)

101.71 (2)

$163.73(5)$

89.04 (3)

$98.58(3)$

131.85 (4) 


\begin{tabular}{|c|c|c|c|}
\hline $\mathrm{O} 2^{\mathrm{vii}}-\mathrm{Sc} 1-\mathrm{K} 1^{\mathrm{viii}}$ & $125.45(2)$ & $\mathrm{P} 2-\mathrm{O} 7-\mathrm{K} 1^{\mathrm{i}}$ & $103.71(3)$ \\
\hline $\mathrm{O} 5^{\mathrm{viii}}-\mathrm{Sc} 1-\mathrm{K} 1^{\mathrm{viii}}$ & $43.401(17)$ & $\mathrm{Sc} 1-\mathrm{O} 7-\mathrm{K} 1^{\mathrm{i}}$ & $114.72(3)$ \\
\hline $\mathrm{O} 1-\mathrm{Sc} 1-\mathrm{K} 1^{\mathrm{viii}}$ & $128.378(19)$ & $\mathrm{P} 2-\mathrm{O} 7-\mathrm{K} 1^{\mathrm{vii}}$ & $102.03(3)$ \\
\hline $\mathrm{O} 7-\mathrm{Sc} 1-\mathrm{K} 11^{\mathrm{viii}}$ & $49.150(18)$ & $\mathrm{Sc} 1-\mathrm{O} 7-\mathrm{K} 1^{\mathrm{viii}}$ & $98.52(2)$ \\
\hline $\mathrm{O}^{\mathrm{v}}-\mathrm{Sc} 1-\mathrm{K} 1^{\mathrm{vi}}$ & $125.03(2)$ & $\mathrm{K} 1^{\mathrm{i}}-\mathrm{O} 7-\mathrm{K} 1^{\mathrm{viii}}$ & $100.37(2)$ \\
\hline $\mathrm{O}^{\mathrm{vi}}-\mathrm{Sc} 1-\mathrm{K} 1^{\mathrm{vi}}$ & $55.89(2)$ & & \\
\hline
\end{tabular}

Symmetry codes: (i) $-x+2,-y+1,-z+2$; (ii) $-x+1, y+1 / 2,-z+3 / 2$; (iii) $-x+2, y+1 / 2,-z+3 / 2$; (iv) $-x+1,-y+1,-z+2$; (v) $x,-y+1 / 2, z+1 / 2$; (vi) $x,-y+1 / 2$, $z-1 / 2$; (vii) $-x+1, y-1 / 2,-z+3 / 2$; (viii) $-x+2, y-1 / 2,-z+3 / 2$. 